\title{
Observational indications of downward-propagating gravity waves in middle atmosphere lidar data
}

\author{
N. Kaifler ${ }^{a}$, B. Kaifler ${ }^{\mathrm{a}}$, B. Ehard ${ }^{\mathrm{a}}$, S. Gisinger ${ }^{\mathrm{a}}$, A. Dörnbrack ${ }^{\mathrm{a}}$, M. Rapp ${ }^{\mathrm{a}}$, \\ R. Kivi ${ }^{\mathrm{b}}$, A. Kozlovsky ${ }^{\mathrm{c}}$, M. Lester $^{\mathrm{d}}$, B. Liley ${ }^{\mathrm{e}}$ \\ ${ }^{a}$ Institute of Atmospheric Physics, German Aerospace Center, Oberpfaffenhofen, \\ Germany \\ ${ }^{b}$ Finnish Meteorological Institute, Sodankylä, Finland \\ ${ }^{c}$ Sodankylä Geophysical Observatory, Finland \\ ${ }^{d}$ University Leicester, United Kingdom \\ ${ }^{e}$ National Institute of Water and Atmospheric Research, Lauder, New Zealand
}

\begin{abstract}
Two Rayleigh lidars were employed at a southern-hemisphere mid-latitude site in New Zealand $\left(45^{\circ} \mathrm{S}\right)$ and a northern-hemisphere high-latitude site in Finland $\left(67^{\circ} \mathrm{N}\right)$ in order to observe gravity waves between 30 and $85 \mathrm{~km}$ altitude under wintertime conditions. Two-dimensional wavelet analysis is used to analyze temperature perturbations caused by gravity waves and to determine their vertical wavelengths and phase progression. In both datasets, upward phase progression waves occur frequently between 30 and $85 \mathrm{~km}$ altitude. Six cases of large-amplitude wave packets are selected which exhibit upward phase progression in the stratosphere and/or mesosphere. We argue that these wave packets propagate downward and we discuss possible wave generation mechanisms. Spectral analysis reveals that superpositions of two or three wave packets are common. Furthermore, their characteristics often match those of upward-propagating waves which are observed at the same time or earlier. In the dataset means, the contribution of upward
\end{abstract}


phase progression waves to the potential energy density $E_{p}$ is largest in the lower stratosphere above Finland. There, $E_{p}$ of upward and downward phase progression waves is comparable. At $85 \mathrm{~km}$ one third of the potential energy carried by propagating waves is attributed to upward phase progression waves. In some cases $E_{p}$ of upward phase progression waves far exceeds $E_{p}$ of downward phase progression waves. The downward-propagating waves might be generated in situ in the middle atmosphere or arise from reflection of upward-propagating waves.

Keywords: Gravity waves, middle atmosphere, lidar, downward-propagating waves

\section{1. Introduction}

$2 \quad$ Atmospheric gravity waves represent an important coupling mechanism 3 between the lower and the middle atmosphere with strong effects on the en4 ergy budget and general circulation of the atmosphere (Holton, 1982). Major 5 sources of gravity waves are flow over topography, convection, spontaneous 6 adjustment and dynamical processes related to the tropospheric jet stream 7 (see Fritts and Alexander, 2003; Plougonven and Zhang, 2014, for reviews of 8 gravity waves).

$9 \quad$ From tropospheric sources, gravity waves propagate horizontally and vertically according to their group velocity which determines the direction of energy propagation. The vertical group velocity is thereby of opposite sign compared to the vertical phase velocity, i.e. downward phase progression means upward energy/wave propagation (Nappo, 2013). The amplitudes of the waves grow with altitude due to the decreasing density. Critical-level 
filtering by the background wind may modify vertical propagation. Breaking of large-amplitude gravity waves in the mesopause region may generate small-scale gravity waves visible, for example, in polar mesospheric clouds (Chandran et al., 2009) and imagery of the airglow layer (Nielsen et al., 2006). Interaction with the mean flow may also cause damping, refraction, reflection and ducting of waves (Fritts and Alexander, 2003). Reflection of primary waves, such as from regions with large vertical gradients of the horizontal wind, results in waves that propagate downward. Idealized model simulations predict the generation of secondary waves by breaking of primary waves in the stratosphere and mesosphere (Satomura and Sato, 1999; Zhou et al., 2002). Downward-propagating gravity waves may also be generated in situ in the middle atmosphere, e.g. in the polar night jet region (Sato and Yoshiki, 2008). These waves, either generated in situ or by reflection of primary waves, may contribute to the downward coupling from the mesosphere to the lower stratosphere.

Downward-propagating waves, i.e. waves with downward energy propagation, were observed in the lower stratosphere by radiosondes at sites inside the polar vortex or close to its edge (Sato and Yoshiki, 2008; Moffat-Griffin et al., 2011; Murphy et al., 2014). Moffat-Griffin et al. (2011) report that up to $60 \%$ of all waves propagate downward and carry about the same amount of energy compared to upward-propagating waves, which is in accordance with the observations by Murphy et al. (2014). There are only few observations in the upper stratosphere and above as radiosondes seldom reach altitudes above $30 \mathrm{~km}$. Wilson et al. (1991) and Yamashita et al. (2009) derived the gravity wave potential energy density of waves with positive ground-based 
vertical phase velocity and thus potentially downward energy propagation at mid- and high-latitude sites from lidar measurements by applying twodimensional Fourier analysis. Wilson et al. (1991) attributed on average one third of the gravity wave energy density to downward-propagating waves in the 30-45 km altitude range at a mid-latitude site, whereas Yamashita et al. (2009) report values up to $50 \%$ in the same altitude range at two Antarctic sites. Model simulations by Sato et al. (2012) also show significant downward energy propagation south of the southern Andes in the lower stratosphere in winter. To the knowledge of the authors, no previous observational studies on downward-propagating gravity waves in the upper mesosphere exist.

We use observational data acquired during two field campaigns dedicated to the study of atmospheric gravity waves. The DEEPWAVE campaign was based at New Zealand during austral winter 2014. Its goal was to quantify gravity wave dynamics and effects from the source regions to the regions of dissipation (Fritts et al., 2016). The GW-LCYCLE2 campaign took place in winter 2015/2016 in northern Scandinavia as part of the Role of the Middle Atmosphere in Climate (ROMIC) programme, with the goal to study dynamical processes in the polar winter atmosphere. Both locations are close to the polar vortex edge where stratospheric wave activity is largest (Whiteway et al., 1997; Baumgaertner and McDonald, 2007). New Zealand is a ,gravity wave hotspot" (Hoffmann et al., 2016), and the Scandinavian Mountains are also known for the occurrence of large-amplitude mountain waves (Dörnbrack et al., 1999). From the global maps of stratospheric gravity wave variances by $\mathrm{Wu}$ and Eckermann (2008), comparable wave activity during winter is inferred for New Zealand and northern Scandinavia. Both campaigns utilized 
comprehensive sets of airborne and ground-based instrumentation including a ground-based lidar. The lidar instruments produced large datasets of highresolution temperature measurements covering the middle atmosphere from 30 up to $85 \mathrm{~km}$ altitude.

In this paper, we apply an advanced spectral analysis method using twodimensional wavelets in order to classify waves based on their vertical phase progression. Downward-propagating wave packets are identified by upward phase progression $c_{z}=\omega / m$, where $\omega$ is the ground-based, observed frequency and $m$ the vertical wavenumber. Regarding this approach, Fritts and Alexander (2003, par. 56) note that for waves with a horizontal phase velocity comparable to and opposed to the mean wind, a false assignment of the direction of energy propagation occurs due to Doppler shifting. Unfortunately, no co-located wind measurements are available at the lidar sites. We utilize meteor radar data and ECMWF model data in our interpretation and give additional arguments that the observed waves are downward-propagating. From both lidar datasets, we estimate the gravity wave background and present selected events in order to characterize downward-propagating gravity waves in the middle atmosphere. These are the first observations of downwardpropagating gravity waves at mesospheric altitudes and we therefore aim to give a thorough description of the observations. Although conclusive evidence and identification of generation processes relies on additional wind information and modelling and is beyond the scope of this paper, we speculate on possible sources and generation mechanisms based on the data at hand in order to stimulate further research.

Instrument details and key figures of the datasets are given in section 2 . 
The analysis involving two-dimensional wavelet transformations is described in section 3. In section 4 the analysis methods are applied to observational data in order to characterize the gravity wave background. Six cases are selected for further investigation. These are described in detail in section 5. In section 6 the interpretation concerning the sources and propagation of the observed wave packets are discussed with respect to the influence of the background wind. A summary is given in section 7 .

\section{Data}

Middle atmospheric temperature measurements obtained by two groundbased Rayleigh lidars are used in this study. The Temperature Lidar for Middle Atmosphere Research (TELMA) and the Compact Rayleigh Autonomous Lidar (CORAL), both recently developed by the German Aerospace Center (DLR), are transportable, semi-autonomous middle atmosphere lidars. Both systems are equipped with pulsed Nd:YAG lasers with $12 \mathrm{~W}$ average power at $532 \mathrm{~nm}$ wavelength and telescopes of $63 \mathrm{~cm}$ diameter for reception of the atmospheric return signal. Two height-cascaded receiver channels are used to increase the dynamic range. From the measured photon count profiles, atmospheric density is inferred and temperature profiles $T(z)$ are obtained by top-down integration assuming hydrostatic equilibrium (Hauchecorne and Chanin, 1980). At the top altitude around $100-110 \mathrm{~km}$, the integration is seeded by SABER temperature measurements. Typical uncertainties in temperature measurements are less than $5 \mathrm{~K}$ at $80 \mathrm{~km}$ and less than $1-2 \mathrm{~K}$ at $60 \mathrm{~km}$ and below for $20 \mathrm{~min}$ resolution. In this paper, we analyze lidar temperature observations in the $30-85 \mathrm{~km}$ altitude range. 
The TELMA instrument was deployed to Lauder, New Zealand $\left(45.0^{\circ} \mathrm{S}\right.$, $169.7^{\circ} \mathrm{E}$ ), during the DEEPWAVE campaign in austral winter 2014 (Fritts et al., 2016; Kaifler et al., 2015). The second dataset was obtained by the CORAL lidar during the GW-LCYCLE2 campaign at Sodankylä, Finland $\left(67.4^{\circ} \mathrm{N}, 26.6^{\circ} \mathrm{E}\right)$, in winter $2015 / 2016$. In total, $1021 \mathrm{~h}$ of atmospheric temperature data are available for analysis. Here, we focus on continuous observations of more than $8 \mathrm{~h}$ duration. This leaves 38 nights $(402 \mathrm{~h})$ from the DEEPWAVE dataset in the period 30 June 2014 - 27 October 2014, and 26 nights (292 h) from GW-LCYCLE2 between 7 October 2015 and 21 March 2016. The two subsets amount to $694 \mathrm{~h}$ in total. The data are organized in time-height matrices with $\Delta t=5 \min \times \Delta z=540 \mathrm{~m}$ resolution. Due to smoothing of raw photon count data in the temperature retrieval, the effective resolution of independent temperature observations is $20 \mathrm{~min} \times$ $2100 \mathrm{~m}$.

\section{Method}

Gravity waves manifest themselves by temperature perturbations $T^{\prime}(z, t)$ superposed on the background temperature profile $T_{0}(z)$. Following Ehard et al. (2015), we extract $T^{\prime}(z, t)$ from the observed temperature $T(z, t)$ by applying a 5th order Butterworth filter with a cutoff wavelength of $15 \mathrm{~km}$. The nightly mean of the background temperature field

$$
T_{0}(z)=\overline{T(z, t)-T^{\prime}(z, t)}
$$

is used to calculate the squared Brunt-Vaisälä frequency $N^{2}(z)$ which represents the ambient atmospheric static stability. Using $T_{0}, T^{\prime}, N^{2}$ and the 
acceleration due to Earth's gravity, $g$, the gravity wave potential energy density normalized by mass

$$
E_{p}(z)=\frac{1}{2} \frac{g^{2}}{N^{2}} \overline{\left(\frac{T^{\prime}}{T_{0}}\right)^{2}}
$$

is derived.

Assuming the temperature perturbations $T^{\prime}$ are caused by a superposition of quasi-monochromatic waves, we decompose the observed gravity wave field using wavelet transformations with two-dimensional Morlet wavelets (Wang and $\mathrm{Lu}, 2010$; Kaifler et al., 2015). The properties of the Morlet wavelet are determined by two parameters: the angle $\theta$, which defines the direction of the harmonic wave in time-height-space ( $t$-z-space), and the scale $s$, which corresponds to the wavelength along this direction. A representation of the 2-d Morlet wavelet in the time/spatial domain is shown in Fig. 1. The parameters $\theta$ and $s$ can be converted to vertical wavelength $\lambda_{z}$ and ground-based period $\tau$ or vertical phase progression

$$
c_{z}=\frac{\omega}{m}=\frac{\lambda_{z}}{\tau}
$$

of a quasi-monochromatic gravity wave. The angle $\theta$ is varied between $0^{\circ}$ (vertical phase lines in $t$ - $z$-space) and $180^{\circ}$ (vertical again) by rotating the wavelet clockwise in steps of 3 deg. The scale $s$ is chosen in a way such that, in case of quasi-stationary waves $\left(\theta=90^{\circ}, c_{z}=0 \mathrm{~m} / \mathrm{s}\right.$, e.g. stationary mountain waves), vertical wavelengths from $2-20 \mathrm{~km}$ are covered. One wavelet transformation is performed for each combination $(\theta, s)$. The spectral power density (i.e. the squared wavelet coefficients scaled by the inverse scale squared, see Liu et al. (2007)), is obtained as a function of $z, t, s\left(\lambda_{z}\right)$ 
and $\theta\left(\tau\right.$ or $\left.c_{z}\right)$. Summation over $z$ and $t$ yields the spectral power as a function of $\lambda_{z}$ and $\tau$ (or $c_{z}$ ), which provides an estimate of the spectral energy distribution of the wave field. Local maxima in those spectrograms indicate dominating wave packets.

From the wavelet coefficients of each transformation the contribution to $T^{\prime}$ from a quasi-monochromatic wave with a particular scale and angle in $t$ $z$-space can be reconstructed. Superposition of all waves restores the original $T^{\prime}$. Waves with upward phase progression $\left(T_{-}^{\prime}\right)$ are reconstructed using the coefficients from all transformations with $\theta>100^{\circ}\left(c_{z}>0.35 \mathrm{~m} / \mathrm{s}\right)$. Conversely, waves with downward phase progression $T_{+}^{\prime}$ are reconstructed from all transformations with $\theta<80^{\circ}\left(c_{z}<-0.35 \mathrm{~m} / \mathrm{s}\right)$.

In analogy to the calculation of $E_{p}$ from $T^{\prime}$, the potential energy densities $E_{p,-}$ and $E_{p,+}$ are estimated from $T_{-}^{\prime}$ and $T_{+}^{\prime}$ as a function of altitude. $E_{p,-}$ $\left(E_{p,+}\right)$ indicates in which altitude ranges waves with upward (downward) phase progression occur. The mean potential energy density profiles $E_{p, 0,-}$ and $E_{p, 0,+}$ are calculated as the dataset mean from 38 nightly mean profiles for the DEEPWAVE dataset and 26 nightly mean profiles for the GWLCYCLE2 dataset, respectively. These values are used to compute energy ratios which give the potential energy density of the selected events with respect to the mean potential energy at the two sites.

\section{Mean spectrograms and case selection}

In order to identify quasi-monochromatic waves that are locally dominant for a given day, we compare the spectral power associated with a particular wave to the power of the mean spectrogram. The latter is computed by aver- 
aging all spectrograms in each of the two datasets. Because the generation of gravity waves and the atmospheric conditions which define their propagation depend on the geographic location and proximity to the polar vortex, we compute the mean spectrograms separately for each dataset (Fig. 2).

The mean spectrograms show the characteristics of gravity waves retained in $T^{\prime}$ which were most prominent during all 38 and 26 nights of measurements for DEEPWAVE and GW-LCYCLE2, respectively. At both sites, stationary gravity waves with $c_{z} \approx 0$, e.g. mountain waves, dominate the mean spectra at a vertical wavelength of 4-12 km (Fig. 2a,b). Slightly enhanced power is found for waves with downward phase progression $\left(c_{z}<0\right)$ compared to upward phase progression waves $\left(c_{z}>0\right)$. This can be seen even clearer in the spectrograms with respect to $\tau$ (Fig. 2c,d). The asymmetry means that downward phase progression waves occur more often or have larger amplitudes and thus carry more energy. In terms of altitude, the largest wavelet power is found at $70-80 \mathrm{~km}$, where wave amplitudes are high.

Using the mean spectrograms as a reference, we compare the spectrograms from all nightly observations to these mean gravity waves by computing differences in spectral power. Positive values in the resulting relative spectrogram indicate wave packets that dominate the wave field in the given case, while negative values suggest that corresponding waves are missing or are at least lower in amplitude. The wave parameters $c_{z}, \lambda_{z}, T$ and $z$ of dominant wave packets can be inferred from the relative spectrograms.

From both lidar datasets, we select six cases for detailed study that stand out in terms of the occurrence of large-amplitude wave packets with positive $c_{z}$ : 14 July 2014 (IOP14) and 25 July 2014 from DEEPWAVE, and 6 De- 
cember 2015, 26 December 2015, 21 January and 16 February 2016 from GW-LCYCLE2. These six cases and their respective properties revealed by the spectral analysis are described in the following section.

\section{Results for six selected cases}

DEEPWAVE (Lauder), 14 July 2014

On 14 July 2014, the atmosphere above Lauder is characterized by a broad stratopause located between 50 and $70 \mathrm{~km}$ with a temperature maximum of only $240 \mathrm{~K}$ (black curve in Fig. 3a). The $N^{2}$ profile (black curve in Fig. 3b) shows an enhancement in static stability at $35-55 \mathrm{~km}$ with a pronouced maximum at $42 \mathrm{~km}$ altitude.

Figure 4a shows the determined temperature perturbations $T^{\prime}$ for this case with the largest wave amplitudes located above $55 \mathrm{~km}$ altitude and strong contributions from upward phase progression waves. Figures $4 \mathrm{~b}, \mathrm{c}$ show the reconstructed $T_{+}^{\prime}$ and $T_{-}^{\prime}$, respectively. Visual comparison of Fig. 4c with Fig. 4a confirms that the analyses method correctly extracted the spectral band of interest. Upward phase progression waves are observed in the whole altitude range between 30 and $80 \mathrm{~km}$, with amplitudes up to $10 \mathrm{~K}$ around $75 \mathrm{~km}$ (Fig. 4c). Both the wave coherence and the high amplitude provide evidence of the existence of upward phase progression waves. Black contours in Figures 4b,c indicate the wavelet power which is large where wave amplitudes are large. Of all cases discussed in this paper, the enhancement in spectral power for upward phase progression waves (i.e. $c_{z}>0$ ) compared to the mean spectrogram is largest for this event. For vertical wavelengths $>7 \mathrm{~km}$ enhancements in spectral power are found for $c_{z} \approx 0.4,1.2$ and 
$2.4 \mathrm{~m} / \mathrm{s}$ (Fig. 4d). The waves with the larger phase progression dominate above $60 \mathrm{~km}$, while the amplitude of the slower waves is larger below (Fig. 4c). $E_{p}$ increases up to $80 \mathrm{~km}$ which indicates that gravity waves can propagate from the lower stratosphere to the upper mesosphere (Fig. 5a). $E_{p}$ closely follows the dataset mean $E_{p, 0}$ (dashed line, Fig. 5a) with the largest deviation detected at $74 \mathrm{~km}$ altitude. This is in accordance with the observations of Pautet et al. (2016) who detected a mountain wave in the OH layer above Auckland island $\left(50^{\circ} \mathrm{S}\right)$ in this night. Pautet et al. (2016) report that the mountain wave encountered instabilities and breaking at the $\mathrm{OH}$ layer altitude. Using ECMWF data, we have verified that background conditions at Lauder and Auckland Island were similar (not shown).

While $E_{p,+}$ is reduced below $55 \mathrm{~km}$ compared to the mean $E_{p, 0,+}$ of the dataset (blue curve in Fig. 6a), $E_{p,-}$ is enhanced (red curve). Between 30 and $60 \mathrm{~km}$, upward phase progression waves dominate over downward phase progression waves (black curve). This is a significant deviation from the dataset means, where downward phase progression waves carry two times more energy than upward phase progression waves. The enhancement of upward phase progression waves coincides with a region of enhanced stability at 35-50 km (black curve in Fig. 3b).

DEEPWAVE (Lauder), 25 July 2014

On 25 July 2014, the stratopause is characterized by a nearly isothermal layer of $245 \mathrm{~K}$ between $38 \mathrm{~km}$ and $50 \mathrm{~km}$ (blue curve in Fig. 3a). Interestingly, the nightly mean temperature profile reveals a deep minimum of $200 \mathrm{~K}$ at $65 \mathrm{~km}$ altitude. The temperature inversion above the mesospheric temperature minimum causes a layer of enhanced stability with $N^{2}$ changing 
from $0.25 \times 10^{-3} \mathrm{~s}^{-2}$ to $0.6 \times 10^{-3} \mathrm{~s}^{-2}$ between 58 and $72 \mathrm{~km}$ (blue curve in Fig. 3b).

The $T^{\prime}$ pattern on 25 July 2014 suggests a strong contribution from quasistationary waves (i.e. horizontally aligned patterns) (Fig. 7a). Spectral analysis confirms a dominance of waves with $c_{z} \approx 0 \mathrm{~m} / \mathrm{s}$ and $\lambda_{z}=10-13 \mathrm{~km}$ (Fig. 7d). Additionally, upward phase progression waves are enhanced after $13 \mathrm{UT}$ around $55 \mathrm{~km}$ with amplitudes of $6 \mathrm{~K}$ (Fig. 7c). Figure $7 \mathrm{f}$ reveals that the vertical wavelength of upward-propagating waves is shifted from higher values around $40 \mathrm{~km}$ to lower values at $60 \mathrm{~km}$.

Stratospheric $E_{p}$ is relatively high and shows no increase above $60 \mathrm{~km}$ (Fig. 5b), suggesting that large-amplitude gravity waves break and/or are being reflected at this altitude level. Below $60 \mathrm{~km}$, large-amplitude upward phase progression waves are strongly enhanced (red curve in Fig. 6b), and they even dominate over downward phase progression waves between 48 and $58 \mathrm{~km}$ (black curve in Fig. 6b). The maximum of $E_{p,+}$ is found at $60 \mathrm{~km}$, coinciding with a rapid increase of $N^{2}$ at this level (Fig. 3b).

\section{GW-LCYCLE2 (Sodankylä), 6 December 2015}

Due to the higher latitude of Sodankylä compared to Lauder, the winter stratopause temperature is generally higher. On 6 December 2015, the temperature maximum of $265 \mathrm{~K}$ is located at $50 \mathrm{~km}$ (red curve in Fig. 3a). The warmer stratopause causes an extended region with significantly reduced stability in the mesosphere, where $N^{2}$ is close to $0.3 \times 10^{-3} \mathrm{~s}^{-2}$ from $51 \mathrm{~km}$ to $66 \mathrm{~km}$ (red curve in Fig. 3b).

Figure 8a shows quasi-stationary waves with maximum amplitudes at $50 \mathrm{~km}$ altitude. Remarkably, upward phase progression waves are found be- 
low $50 \mathrm{~km}$ and downward phase progression waves above. Downward phase progression waves have largest temperature perturbations around $55 \mathrm{~km}$ at 15 UT and 21 UT as indicated by the black contour lines in Fig. 8b. Shortly after, the spectral power associated with upward phase progression waves maximizes at slightly lower altitudes, at $50 \mathrm{~km}$ at $16 \mathrm{UT}$ and at $45 \mathrm{~km}$ at 22 UT (Fig. 8c). Vertical wavelengths of downward and upward phase progression waves at $\sim 50 \mathrm{~km}$ altitude are in the same range $(10-12 \mathrm{~km}$, Fig. 8f).

$E_{p}$ is enhanced in the stratosphere between 40 and $60 \mathrm{~km}$ and a second peak that can be ascribed to downward phase progression waves occurs in the upper mesosphere (Fig. 5c and 6c). Maximum wave amplitudes are observed above $80 \mathrm{~km}$ between 15-16 UT and 19-22 UT (Fig. 8b). Towards the end of the observation, around $22 \mathrm{UT}$, large-amplitude upward phase progression waves occur around $80 \mathrm{~km}$, i.e. below the maximum of downward phase progression waves (Fig. 8c).

\section{GW-LCYCLE2 (Sodankylä), 26 December 2015}

On 26 December 2015, the stratopause is found at $48 \mathrm{~km}$ reaching a maximum temperature of $280 \mathrm{~K}$ (orange curve in Fig. 3a). The stability is large below the stratopause and reduced in the region above between 50 and $65 \mathrm{~km}$, followed by a relatively strong increase of $N^{2}$ above $65 \mathrm{~km}$ (Fig. 3b). Figure 9a reveals dominant downward phase progression waves on this day, however the coherent pattern is disturbed by interference with waves of smaller amplitude, e.g. around $40 \mathrm{~km}$ altitude between $22 \mathrm{UT}$ and 5 UT. Downward phase progression waves with amplitudes up to $10 \mathrm{~K}$ are observed in most of the middle atmosphere (Fig. 9b and blue curve in Fig. 6d). 
Two distinct types of upward phase progression waves seem to be superposed (Fig. 9c). The first group has a large vertical wavelength $(12 \mathrm{~km})$, a low phase progression and is confined to altitudes below $55 \mathrm{~km}$. The second group comprises waves with very high vertical phase progression $(\approx 2.5 \mathrm{~m} / \mathrm{s})$ that are visible in the whole altitude range $30-85 \mathrm{~km}$ with temperature perturbations up to $5 \mathrm{~K}$ above $65 \mathrm{~km}$ (Fig. 9c).

Stratospheric $E_{p}$ is strongly enhanced, compared to the dataset mean, and maximizes shortly above the stratopause, meaning that gravity wave amplitudes are large and do not increase further with altitude above (Fig. 5d). A local minimum in $E_{p,+}$ occurs at $64 \mathrm{~km}$ altitude (blue curve in Fig. 6d). Upward phase progression waves are enhanced below $72 \mathrm{~km}$ and reach their maximum amplitudes at $38 \mathrm{~km}$, where $E_{p,-}$ is about a factor of 3.5 above the background (red curve in Fig. 6d). The point of peak $E_{p,+}(52 \mathrm{~km}$ altitude) is located above the altitude where $E_{p,-}$ maximizes (38 km, Fig. $6 \mathrm{f}$ ).

GW-LCYCLE2 (Sodankylä), 21 January 2016

On 21 January 2016, the stratopause is located at $42 \mathrm{~km}$ (brown curve in Fig. 3a) and therefore lowest among the six cases discussed in this study. The mesospheric temperature minimum is found at $70 \mathrm{~km}$.

The relative spectrogram reveals that quasi-stationary waves are mostly absent and the spectrum is dominated by wave packets with downward and upward phase progression and high vertical phase velocities and vertical wavelengths $>5 \mathrm{~km}$ (Fig. 10d). Their superposition creates the strong interference pattern in the $T^{\prime}$ display, e.g. between 45 and $65 \mathrm{~km}$, from $16 \mathrm{UT}$ to 0 UT (Fig. 10a). Two local maxima of upward phase progression waves are found at $62 \mathrm{~km}$ and $17 \mathrm{UT}$ and around $50 \mathrm{~km}$ and 0 UT (Fig. 10c). Smaller- 
scale waves with short wavelength and high vertical phase progression occur in the altitude range $55-85 \mathrm{~km}$ (Fig. 10c).

$E_{p,+}$ steadily increases with altitude maximizing at $65-70 \mathrm{~km}$ (Fig. 6e). The maximum coincides with an enhancement in spectral power of downward phase progressing waves around 0 UT (Fig. 10b). Upward phase progression waves are enhanced above $40 \mathrm{~km}$ (red curve in Fig. 6e). Below $60 \mathrm{~km}$, they even dominate over downward phase progression waves (black curve).

\section{GW-LCYCLE2 (Sodankylä), 16 February 2016}

On 16 February 2016, the stratopause is located at $43 \mathrm{~km}$ with a temperature of $260 \mathrm{~K}$ (purple curve in Fig. 3a). Above the stratopause the temperature decreases very slowly up to $60 \mathrm{~km}$ altitude, and the mesospheric temperature minimum is located above $80 \mathrm{~km}$. This leads to an almost constant static stability of $0.35 \times 10^{-3} \mathrm{~s}^{-2}$ between 47 and $67 \mathrm{~km}$ and a slow increase of $N^{2}$ above $67 \mathrm{~km}$ (Fig. 3b).

On this day, quasi-stationary waves are strongly suppressed (Fig. 11d). Figures 11b and 11c show a clear partition with $T_{+}^{\prime}$ and $T_{-}^{\prime}$ being enhanced in the mesosphere and stratosphere, respectively. The relative spectrogram is mostly symmetric and reveals wave groups with phase velocities of $0.2,0.9$ and $2 \mathrm{~m} / \mathrm{s}$ (Fig. 11d).

$E_{p}$ is enhanced around $47 \mathrm{~km}$ and $74 \mathrm{~km}$, where the upper maximum is caused by downward phase progression waves, and the lower one by upward phase progression waves (Fig. $5 \mathrm{f}$ and $6 \mathrm{f}$ ), in agreement with the results of the spectral analysis. 


\section{Discussion}

First, we assess the question whether gravity waves with observed upward phase progression (Fig. 4,7-11c) are indeed transporting energy downward. We estimate the intrinsic horizontal phase velocity $\left|\hat{c}_{h}\right|$ of downwardpropagating waves using the mid-frequency approximation of the gravity wave dispersion relation (eqn. 33, Fritts and Alexander, 2003)

$$
|m|=\frac{N}{\left|\hat{c}_{h}\right|}
$$

which is valid for the waves observed here. $N$ is thereby taken from Fig. 3b.

The vertical wavenumber $m$ is estimated via the relation $m=\frac{2 \pi}{\lambda_{z}}$ from $\lambda_{z}$ as indicated by the black curves in Fig. 4,7-11f. Here, $\lambda_{z}$ was averaged over all $\lambda_{z}>0$ derived from the perturbations of upward phase progression waves shown in Fig. 4,7-11c. $\hat{c}_{h}$ is related to the background wind $\bar{u}_{h}$ through

$$
\hat{c}_{h}=c_{h}-\bar{u}_{h}
$$

$\hat{c}_{h}$ and $c_{h}$ are of opposite sign if the condition

$$
\hat{c}_{h}<-\bar{u}_{h}
$$

is fulfilled, i.e. for intrinsic phase velocities $\left|\hat{c}_{h}\right|$ smaller and opposed to the background wind. In this case, $m$ and $c_{z}$ change sign and thus upwardpropagating waves would appear as upward phase progression waves to a ground-based lidar. A detailed analysis of the influence of Doppler shifting on vertical phase progressions as observed by ground-based lidars is given by Dörnbrack et al. (2017). 
A full assessment of the direction of energy propagation of upward phase progression waves is hindered by two limitations: (1) the horizontal propagation direction of the waves and thus the angle between $\hat{c}_{h}$ and $\bar{u}_{h}$ is unknown, and (2) no co-located wind measurements are available at Lauder and Sodankylä in the relevant altitude range. We assume that the horizontal propagation of waves is aligned with the background wind as a worst-case scenario. For Sodankylä, we utilize radar winds above $82 \mathrm{~km}$. Below $60 \mathrm{~km}$, ECMWF horizontal winds serve as an estimate. Le Pichon et al. (2015) showed that ECMWF winds are in agreement with co-located wind radiometer measurements up to $40 \mathrm{~km}$. ECMWF wind data were extracted for the locations of the lidar sites and averaged over the lidar measurement time (Fig. 12a).

Typical values of $\left|\hat{c_{h}}\right|$ lie in the range $30-50 \mathrm{~m} / \mathrm{s}$ while horizontal winds are typically larger than $30 \mathrm{~m} / \mathrm{s}$ (Fig. 12). In two cases, maximum wind speeds of $110 \mathrm{~m} / \mathrm{s}$ are reached. Based on the data at hand, it is therefore not possible to prove that the observed positive ground-based vertical phase progression is not a result of the waves being strongly Doppler shifted in all cases. However, several arguments speak for downward-propagating waves.

A false assignment of vertical directionality of energy propagation due to Doppler shifting is expected to occur primarily at altitudes where the horizontal wind speed is maximum, but not over the whole altitude range analyzed in Fig. 4,7-11c. On 14 July 2014, waves with upward phase progression are detected between 30 and $80 \mathrm{~km}$ (Fig. 4c). The occurrence of coherent phase lines over this deep altitude range is therefore an indication for downward-propagating waves. Then, the source of these waves must be located in the upper mesosphere or lower thermosphere. As suggested by 
Pautet et al. (2016), it is possible that the source is the breaking of a mountain wave. Waves with large positive phase progression are also visible across the altitude range 30-85 km on 26 December 2015 (Fig. 9c).

Furthermore, at altitudes above $80 \mathrm{~km}$, the wind speed can be expected to be low, making Doppler shifting less likely. Yet, in some cases occurrences of large-amplitude waves with upward phase progression are detected at altitudes around $80 \mathrm{~km}$, e.g. on 14 July 2014, 6 December 2015, 26 December 2015 and 21 January 2016. For Sodanyklä, meteor radar wind observations are available. On 6 December 2015, horizontal phase velocities of $\left|\hat{c}_{h}\right|>45 \mathrm{~m} / \mathrm{s}$ are signficantly larger than the measured wind speed of $30 \mathrm{~m} / \mathrm{s}$. In this case it is therefore safe to assume that the observed wave packet propagates downward. This means at the same time that the source of these waves must be located above the altitude of their occurrence, i.e. in the mesopause region or lower thermosphere. A possible source is secondary gravity waves, whose generation in the thermosphere was proposed by Vadas and Liu (2009).

On 25 July 2014, the maximum amplitude of downward-propagating waves occurs in the range 45-63 km (Fig. 6b), above the wind maximum which is found at $43 \mathrm{~km}$ (Fig. 12a). The shortening vertical wavelength of upward-propagating waves visible in Fig. $7 \mathrm{f}$ is evidence for partial reflection of waves at the bottom of the mesospheric inversion layer (Fig. 3a). The occurrence of downward-propagating waves below $65 \mathrm{~km}$ might therefore arise from reflection or partial reflection of upward-propagating waves, or from the breaking of these waves.

In case of large Doppler shifting, one would expect waves of all vertical 
phase velocities to be shifted in one direction, thus the spectrograms would not be symmetric. Yet the spectrograms in Fig. 8d-f on 6 December 2015 are remarkably symmetric with respect to $c_{z}, \tau$ and $\lambda_{z}$, respectively. This means that for every wave packet with upward phase progression, also a wave packet with downward phase progression of similar vertical wavelength is observed. This duality appears not only to be valid for individual wave packets, but also holds for the mean spectrograms shown in Fig. 2. Sato and Yoshiki (2008) observe a similar relationship between wave parameters of upward and downward-propagating waves in radiosonde data and conclude that the waves must originate from the same source.

Comparison of the horizontal phase velocities on 6 December 2015 with ECMWF horizontal wind (red curve in Fig. 12a) shows comparable velocities at two altitudes, $45 \mathrm{~m} / \mathrm{s}$ at $40 \mathrm{~km}$ and $35 \mathrm{~m} / \mathrm{s}$ at $57 \mathrm{~km}$. At both altitudes large-amplitude wave packets with upward vertical phase velocities exist, and they are therefore very likely downward-propagating waves. We want to emphasize here that the observed wave packet shows slanted phase lines in the height-time cross section. If the positive sign of the vertical phase speed were to be caused by Doppler shifting, the horizontal wind speed would have to be much larger than the intrinsic phase speed, which is not the case here. A possible source of the wave packet is reflection of waves propagating from below into the region of reduced stability above the stratopause. A similar situation is found on 26 December 2015, when the largest temperature perturbations of downward-propagating waves are found below $40 \mathrm{~km}$ altitude, i.e. below the wind maximum.

The observation on 16 February 2016 takes on a special position. Here 
the maxima of $E_{p,-}$ at $48 \mathrm{~km}$ and $E_{p,+}$ at $73 \mathrm{~km}$ are separated by $25 \mathrm{~km}$ in altitude (Fig. 6f). Close inspection of Figs. 11b and c reveal that maxima in spectral power are also well separated and are subject to similar temporal evolution. The contour lines associated with spectral power of upwardpropagating waves extend to lower altitudes as time progresses, from $70 \mathrm{~km}$ at $18 \mathrm{UT}$ to $45 \mathrm{~km}$ at $2 \mathrm{UT}$ (Fig. 11b). At the same time, the spectral power of downward-propagating waves shifts also to lower altitudes, from $60 \mathrm{~km}$ at $22 \mathrm{UT}$ to $50 \mathrm{~km}$ at $2 \mathrm{UT}$ (Fig. 11c). The simultaneous appearance points to a common source which changes its characteristics over time. It seems possible that both upward and downward-propagating waves are generated in situ within the same volume between $50 \mathrm{~km}$ and $65 \mathrm{~km}$ altitude. A likely candidate mechanism is spontaneous adjustment near the edge of the polar vortex. Indeed, polar maps of ECMWF geopotential height and potential vorticity show that Sodankylä is situated just at the inner edge of the polar vortex on this day (not shown here).

The different background conditions throughout the six cases shows that generation and propagation of downward-propagating waves are not tied to a specific atmospheric situation. Indeed, the mean spectrograms in Fig. 2 show that their occurrence is a common feature in the middle atmosphere and is not restricted to single observations. We showed in this study that local maxima in potential energy densities associated with downward-propagating waves are often accompanied by maxima of upward-propagating waves. The maxima associated with downward-propagating waves are 2-12 km lower in altitude (compare blue and red curves in Fig. 6). This suggests that part of the energy which is carried by upward-propagating waves is transferred 
to downward-propagating waves. Possible mechanisms are partial reflection of primary waves as well as wave breaking with subsequent generation of secondary waves. The downward-propagating waves interfere with other downward-propagating waves which have been generated at lower altitudes, either through reflection or by in situ processes e.g. spontaneous adjustment. Hence, the ratio $E_{p,-} / E_{p,+}$ should increase with decreasing altitude. We note that this simplified theoretical consideration is based on the assumption that downward-propagation is conservative which is certainly not fulfilled in all cases. Our data show nevertheless the expected behaviour: In the GWLCYCLE2 dataset $E_{p, 0,-} / E_{p, 0,+}$ increases from 0.3 at $75 \mathrm{~km}$ altitude to 1.1 at $30 \mathrm{~km}$ (dashed curves in Fig. 6c-f). The energy ratio of approximately 1, which we observed in the mid stratosphere, is consistent with several studies at sites in Antarctica where stratospheric $E_{p,-}$ and $E_{p,+}$ was estimated from radiosonde data (e.g. Moffat-Griffin et al., 2011). A ratio of $\approx 0.5$ was reported in a lidar-based study of stratospheric gravity waves at mid latitudes (Wilson et al., 1991). We note that the observed energy ratio may depend on geographic latitude and the location of the observation relative to the polar vortex. For Lauder, a mid-latitude site, we find a value of 0.6 for the mid stratosphere. Moreover, downward-propagating large-amplitude waves occur less frequently compared to the observations at Sodankylä (dashed curves in Fig. $6 \mathrm{a}$ and c). A commonality of both datasets is an increase of $E_{p, 0,-} / E_{p, 0,+}$ with increasing altitude above $75 \mathrm{~km}$. The larger energy ratio in the upper mesosphere may be indicative of a source of downward-propagating waves located in the lower thermosphere. On average, downward-propagating waves contribute up to about one third to the energy flux carried by propagating 
waves at $80 \mathrm{~km}$ altitude.

The identification of wave sources and generation processes must remain speculative without co-located high-resolution wind measurements. Advances in lidar technology might allow for high-resolution common-volume wind soundings in the future (Baumgarten et al., 2015). Background winds not only cause Doppler shifting but also have a large impact on wave propagation. For example, wind shears cause refraction of waves, and vertical propagation of waves can be prevented by wind-induced critical levels.

Further limitations result from possible ambiguity in data interpretation. In addition to gravity wave sources, local maxima in potential energy density profiles can also be caused by oblique-propagating waves and trapped waves. Conversely, a minimum in $E_{p}$ may not necessarily be indicative of wave dissipation at this altitude. However, as shown in this study, downwardpropagating waves usually extend over a large altitude range, often covering the whole range from $30 \mathrm{~km}$ to $85 \mathrm{~km}$. This suggests that wave packets are sufficiently large in space and time so that oblique-propagating waves on average do not cause localized maxima in $E_{p}$ profiles. The size of the wave packets and the direction of horizontal wave propagation direction could be inferred from multi-site lidar observations with a suitable geometry.

\section{Conclusions}

In this paper, we study wave packets with upward phase progression in the middle atmosphere above two sites close to the Arctic and Antarctic polar vortex edge, respectively. The high-resolution measurements were obtained using two Rayleigh lidars situated in Lauder, New Zealand and 
Sodankylä, Finland. Propagating waves were extracted from measured temperature profiles in the altitude range $30-85 \mathrm{~km}$ by means of two-dimensional wavelet analysis. This method allows us to determine vertical wavelengths and ground-based vertical phase velocities. Our observations show that wave packets with upward phase progression are commonly observed in the stratosphere and mesosphere. Those are identified with downward-propagating waves for negligible Doppler shifting by the background wind. We selected six cases with large-amplitude wave packets which were presented and discussed in detail. Although conclusive evidence relies on additional measurements yet unavailable, we give several arguments that those waves are indeed downward-propagating.

From both our datasets, the contribution of downward-propagating waves in terms of potential energy density is highest in the stratosphere above Sodankylä $\left(67^{\circ} \mathrm{N}\right)$. About one third of the potential energy density at $85 \mathrm{~km}$ altitude is carried by downward-propagating waves. Often, maxima in potential energy density of upward-propagating waves are observed at or below layers of enhanced stability in the atmosphere and are accompanied by maxima of downward-propagating waves $2-12 \mathrm{~km}$ below. We speculate that in these cases downward-propagating waves are reflected primary waves. Vertical wavelengths and phase progressions inferred from spectrograms are comparable for both up- and downward-propagating waves, indicating that the waves originate from a common source. In one case, up- and downward-propagating waves appear to be generated in situ within the same volume between $50 \mathrm{~km}$ and $65 \mathrm{~km}$ altitude. Such upward-propgating waves from sources in the middle atmosphere might be of relevance for mesosphere-ionosphere coupling. A 
likely generation mechanism is spontaneous adjustment near the edge of the polar vortex. However, the generation mechanism cannot be determined with certainty based on lidar observations alone. Model simulations are required e.g. to establish boundary conditions for the reflection of gravity waves.

\section{Acknowledgements}

We thank the PIs of DEEPWAVE and GW-LCYCLE2 for the joint effort during the field campaigns. The authors thank H. Wilms for assistance with calculations. N. Kaifler was supported by the Helmholtz association in the project PD-206. B. Ehard and S. Gisinger were supported through the projects Processes And Climatology Of Gravity Waves (PACOG, RA 1400/61) and Modification of gravity waves propagating across the tropopause (GWTP,DO 1020/9-1) in the framework of the research unit Multi-scale Dynamics of Gravity Waves (MS-GWaves) funded by the German Research Foundation. Campaigns were partly funded by the German Ministry of Education and Research $(\mathrm{BMBF})$ in the priority programme Role Of the Middle atmosphere In Climate (ROMIC). SABER temperature data was taken from the webpage. ECMWF is acknowledged for providing wind profiles. We thank four anonymous reviewers for their comments and suggestions which helped us to improve the manuscript.

Baumgaertner, A. J. G., McDonald, A. J., 2007. A gravity wave climatology for Antarctica compiled from challenging minisatellite payload/global positioning system (CHAMP/GPS) radio occultations. Journal of Geophysical Research: Atmospheres 112 (D5), n/a-n/a, d05103.

URL http://dx.doi.org/10.1029/2006JD007504 
Baumgarten, G., Fiedler, J., Hildebrand, J., Lbken, F.-J., 2015. Inertia gravity wave in the stratosphere and mesosphere observed by doppler wind and temperature lidar. Geophysical Research Letters 42 (24), 10,929-10,936, 2015 GL066991.

URL http://dx.doi.org/10.1002/2015GL066991

Chandran, A., Rusch, D., Palo, S., Thomas, G., Taylor, M., 2009. Gravity wave observations in the summertime polar mesosphere from the cloud imaging and particle size (CIPS) experiment on the AIM spacecraft. Journal of Atmospheric and Solar-Terrestrial Physics 71 (34), 392 400, global Perspectives on the Aeronomy of the Summer Mesopause RegionEighth International Workshop on Layered Phenomena in the Mesopause Region.

URL http://www.sciencedirect.com/science/article/pii/S1364682608002745

Dörnbrack, A., Gisinger, S., Kaifler, B., 2017. On the interpretation of gravity wave measurements by ground-based lidars. Atmosphere 8 (3).

URL http: //www.mdpi.com/2073-4433/8/3/49

Dörnbrack, A., Leutbecher, M., Kivi, R., Kyrö, E., 1999. Mountain-waveinduced record low stratospheric temperatures above northern Scandinavia. Tellus A 51 (5), 951-963.

URL http://dx.doi.org/10.1034/j.1600-0870.1999.00028.x

Ehard, B., Kaifler, B., Kaifler, N., Rapp, M., 2015. Evaluation of methods for gravity wave extraction from middle-atmospheric lidar temperature measurements. Atmospheric Measurement Techniques 8 (11), 4645-4655. URL http: //www . atmos-meas-tech .net/8/4645/2015/ 
Fritts, D. C., Alexander, M. J., 2003. Gravity wave dynamics and effects in the middle atmosphere. Reviews of Geophysics 41 (1), n/a-n/a, 1003.

URL http://dx.doi.org/10.1029/2001RG000106

Fritts, D. C., Smith, R. B., Taylor, M. J., Doyle, J. D., Eckermann, S. D., Dörnbrack, A., Rapp, M., Williams, B. P., Pautet, P.-D., Bossert, K., Criddle, N. R., Reynolds, C. A., Reinecke, P. A., Uddstrom, M., Revell, M. J., Turner, R., Kaifler, B., Wagner, J. S., Mixa, T., Kruse, C. G., Nugent, A. D., Watson, C. D., Gisinger, S., Smith, S. M., Lieberman, R. S., Laughman, B., Moore, J. J., Brown, W. O., Haggerty, J. A., Rockwell, A., Stossmeister, G. J., Williams, S. F., Hernandez, G., Murphy, D. J., Klekociuk, A. R., Reid, I. M., Ma, J., 2016. The deep propagating gravity wave experiment (deepwave): An airborne and ground-based exploration of gravity wave propagation and effects from their sources throughout the lower and middle atmosphere. Bulletin of the American Meteorological Society 97 (3), 425-453.

URL http://dx.doi.org/10.1175/BAMS-D-14-00269.1

Hauchecorne, A., Chanin, M.-L., 1980. Density and temperature profiles obtained by lidar between 35 and $70 \mathrm{~km}$. Geophysical Research Letters 7 (8), $565-568$.

URL http://dx.doi.org/10.1029/GL007i008p00565

Hoffmann, L., Grimsdell, A. W., Alexander, M. J., 2016. Stratospheric gravity waves at southern hemisphere orographic hotspots: 2003-2014 airs/aqua observations. Atmospheric Chemistry and Physics 16 (14), 9381- 
9397.

URL http: //www .atmos-chem-phys . net/16/9381/2016/

Holton, J. R., 1982. The role of gravity wave induced drag and diffusion in the momentum budget of the mesosphere. Journal of the Atmospheric Sciences 39 (4), 791-799.

URL http: //dx.doi .org/10.1175/1520-0469(1982)039<0791: TROGWI>2 . 0 . C0;2

Kaifler, B., Kaifler, N., Ehard, B., Dörnbrack, A., Rapp, M., Fritts, D. C., 2015. Influences of source conditions on mountain wave penetration into the stratosphere and mesosphere. Geophysical Research Letters 42 (21), 9488-9494, 2015GL066465.

URL http://dx.doi .org/10.1002/2015GL066465

Le Pichon, A., Assink, J. D., Heinrich, P., Blanc, E., Charlton-Perez, A., Lee, C. F., Keckhut, P., Hauchecorne, A., Rüfenacht, R., Kämpfer, N., Drob, D. P., Smets, P. S. M., Evers, L. G., Ceranna, L., Pilger, C., Ross, O., Claud, C., 2015. Comparison of co-located independent ground-based middle atmospheric wind and temperature measurements with numerical weather prediction models. Journal of Geophysical Research: Atmospheres 120 (16), 8318-8331, 2015JD023273.

URL http://dx.doi.org/10.1002/2015JD023273

Liu, Y., Liang, X. S., Weisberg, R. H., 2007. Rectification of the bias in the wavelet power spectrum. Journal of Atmospheric and Oceanic Technology 24 (12), 2093-2102.

URL http://dx.doi.org/10.1175/2007JTECH0511.1 
Moffat-Griffin, T., Hibbins, R. E., Jarvis, M. J., Colwell, S. R., 2011. Seasonal variations of gravity wave activity in the lower stratosphere over an Antarctic Peninsula station. Journal of Geophysical Research: Atmospheres 116 (D14), n/a-n/a, d14111.

URL http://dx.doi.org/10.1029/2010JD015349

Murphy, D. J., Alexander, S. P., Klekociuk, A. R., Love, P. T., Vincent, R. A., 2014. Radiosonde observations of gravity waves in the lower stratosphere over Davis, Antarctica. Journal of Geophysical Research: Atmospheres 119 (21), 11,973-11,996.

URL http://dx.doi.org/10.1002/2014JD022448

Nappo, C. J., 2013. An introduction to atmospheric gravity waves. Academic Press.

Nielsen, K., Taylor, M. J., Pautet, P.-D., Fritts, D. C., Mitchell, N., Beldon, C., Williams, B. P., Singer, W., Schmidlin, F. J., Goldberg, R. A., 2006. Propagation of short-period gravity waves at high-latitudes during the MaCWAVE winter campaign. Annales Geophysicae 24 (4), 1227-1243. URL http://www . ann-geophys . net/24/1227/2006/

Pautet, P.-D., Taylor, M. J., Fritts, D. C., Bossert, K., Williams, B. P., Broutman, D., Ma, J., Eckermann, S. D., Doyle, J. D., 2016. Large-amplitude mesospheric response to an orographic wave generated over the southern ocean auckland islands (50.7s) during the deepwave project. Journal of Geophysical Research: Atmospheres 121 (4), 1431-1441, 2015JD024336. URL http://dx.doi .org/10.1002/2015JD024336 
Plougonven, R., Zhang, F., 2014. Internal gravity waves from atmospheric jets and fronts. Reviews of Geophysics 52 (1), 33-76.

URL http: //dx.doi.org/10.1002/2012RG000419

Sato, K., Tateno, S., Watanabe, S., Kawatani, Y., 2012. Gravity wave characteristics in the southern hemisphere revealed by a high-resolution middleatmosphere general circulation model. Journal of the Atmospheric Sciences 69 (4), 1378-1396.

URL http://dx.doi.org/10.1175/JAS-D-11-0101.1

Sato, K., Yoshiki, M., 2008. Gravity wave generation around the polar vortex in the stratosphere revealed by 3-hourly radiosonde observations at Syowa station. Journal of the Atmospheric Sciences 65 (12), 3719-3735.

URL http://dx.doi.org/10.1175/2008JAS2539.1

Satomura, T., Sato, K., 1999. Secondary generation of gravity waves associated with the breaking of mountain waves. Journal of the Atmospheric Sciences 56 (22), 3847-3858.

URL http://dx.doi .org/10.1175/1520-0469(1999) 056<3847:SGOGWA>2 . 0.CO;2

Vadas, S. L., Liu, H.-l., 2009. Generation of large-scale gravity waves and neutral winds in the thermosphere from the dissipation of convectively generated gravity waves. Journal of Geophysical Research: Space Physics 114 (A10), n/a-n/a, a10310.

URL http://dx.doi.org/10.1029/2009JA014108

Wang, N., Lu, C., 2010. Two-dimensional continuous wavelet analysis and its application to meteorological data. Journal of Atmospheric and Oceanic 
Technology 27 (4), 652-666.

URL http://dx.doi .org/10.1175/2009JTECHA1338.1

Whiteway, J. A., Duck, T. J., Donovan, D. P., Bird, J. C., Pal, S. R., Carswell, A. I., 1997. Measurements of gravity wave activity within and around the arctic stratospheric vortex. Geophysical Research Letters 24 (11), $1387-1390$.

URL http://dx.doi.org/10.1029/97GL01322

Wilson, R., Chanin, M. L., Hauchecorne, A., 1991. Gravity waves in the middle atmosphere observed by Rayleigh lidar: 1. case studies. Journal of Geophysical Research: Atmospheres 96 (D3), 5153-5167.

URL http://dx.doi.org/10.1029/90JD02231

Wu, D. L., Eckermann, S. D., 2008. Global gravity wave variances from Aura MLS: Characteristics and interpretation. Journal of the Atmospheric Sciences 65 (12), 3695-3718.

URL http://dx.doi.org/10.1175/2008JAS2489.1

Yamashita, C., Chu, X., Liu, H.-L., Espy, P. J., Nott, G. J., Huang, W., 2009. Stratospheric gravity wave characteristics and seasonal variations observed by lidar at the South Pole and Rothera, Antarctica. Journal of Geophysical Research: Atmospheres 114 (D12), n/a-n/a, d12101.

URL http://dx.doi.org/10.1029/2008JD011472

Zhou, X., Holton, J. R., Mullendore, G. L., 2002. Forcing of secondary waves by breaking of gravity waves in the mesosphere. Journal of Geophysical 


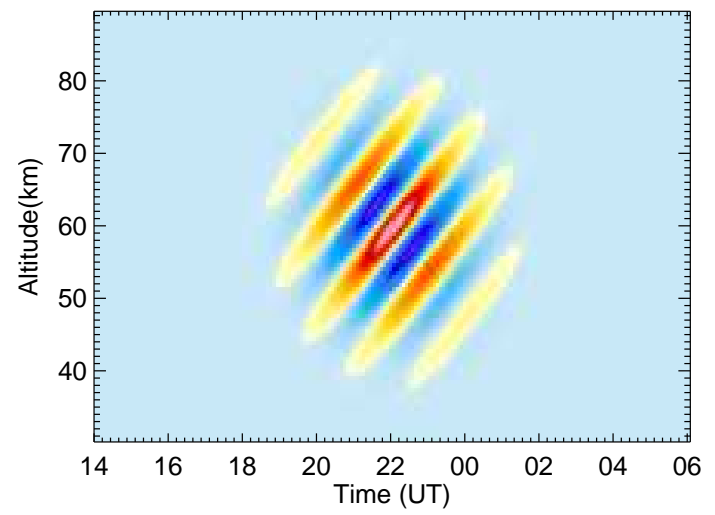

Figure 1: Representation of the 2-d Morlet wavelet in the spatial/time domain with $s=$ $14.3, \theta=135^{\circ}$, corresponding to a vertical wavelength $\lambda_{z}=13 \mathrm{~km}$ and upward phase progression by $c_{z}=2 \mathrm{~m} / \mathrm{s}$.

Research: Atmospheres 107 (D7), ACL 3-1-ACL 3-7.

708

URL http://dx.doi .org/10.1029/2001JD001204 

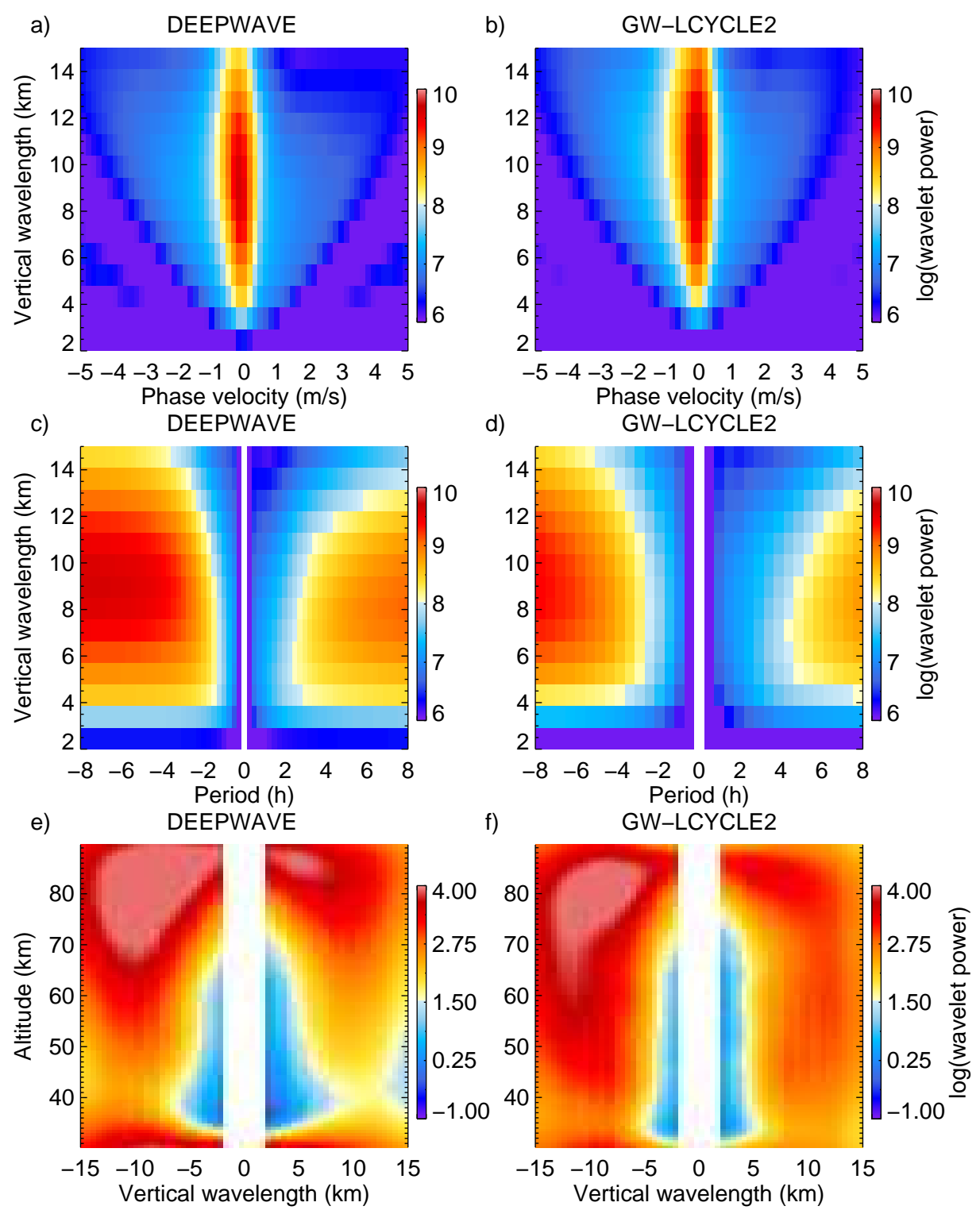

Figure 2: Mean spectrograms of the two datasets: (left) DEEPWAVE, Lauder, (right) GW-LCYCLE2, Sodankylä. Spectral power is shown as a function of $(\mathrm{a}, \mathrm{b}) \lambda_{z}$ vs. $c_{z},(\mathrm{c}, \mathrm{d})$ $\lambda_{z}$ vs. $\tau$ and (e,f) $z$ vs. $\lambda_{z}$. 

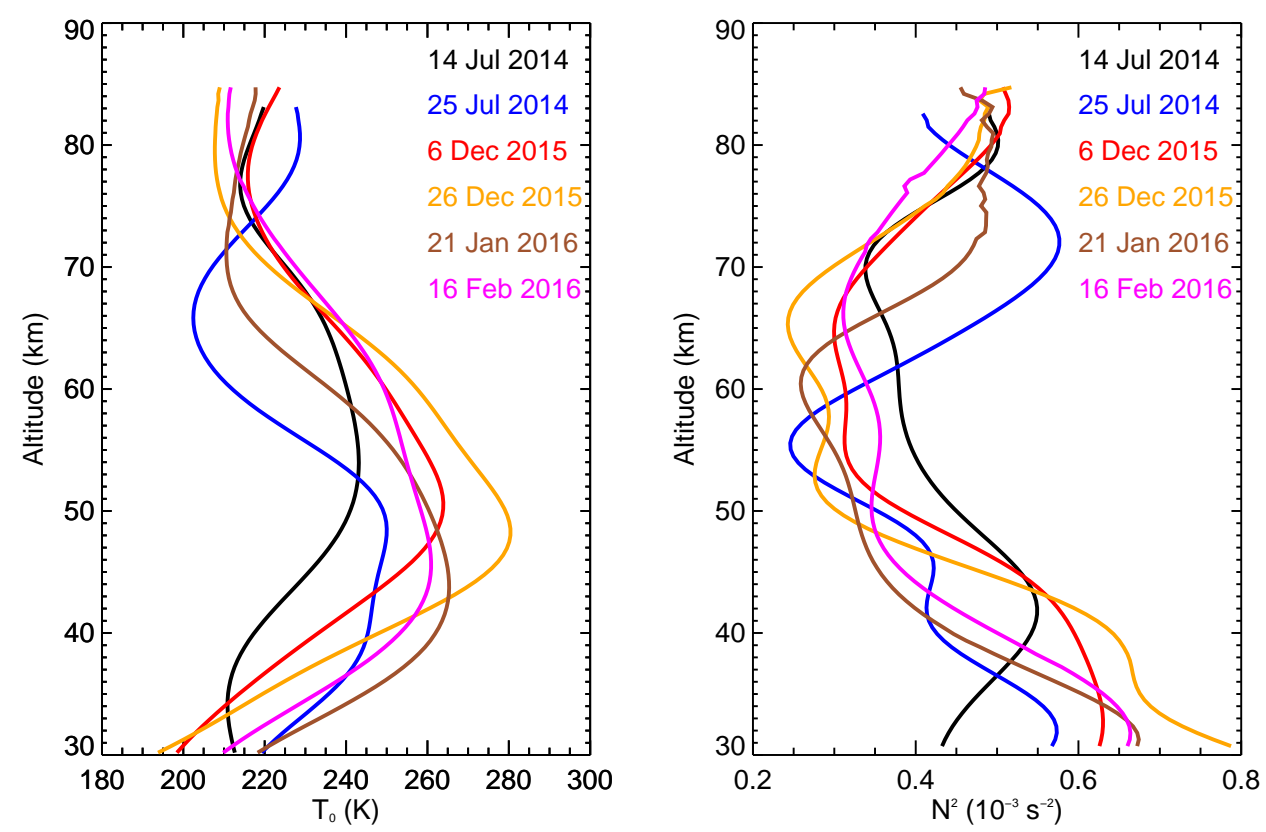

Figure 3: (a) Background temperature $T_{0}$ and (b) atmospheric stability $N^{2}$ for the six selected cases. 
a)
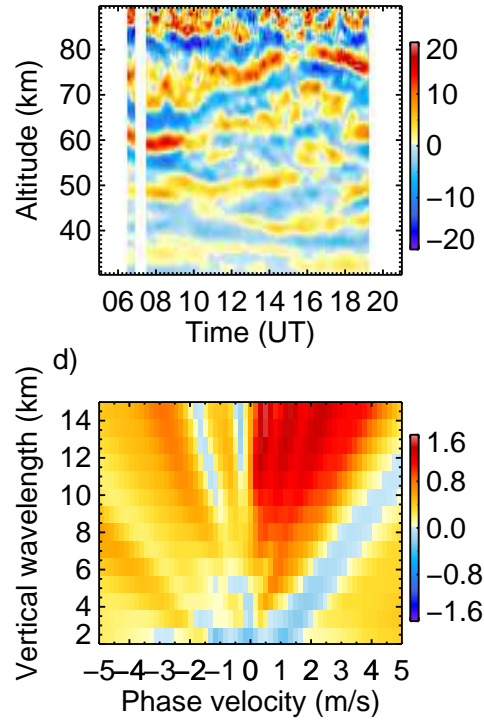

b)

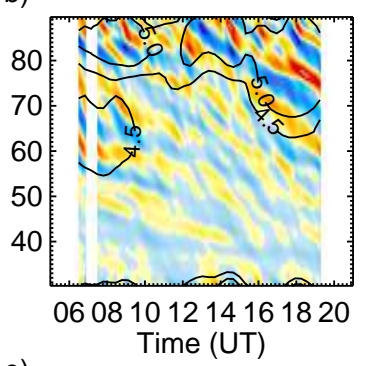

e)

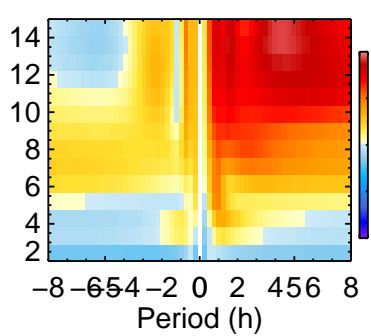

c)

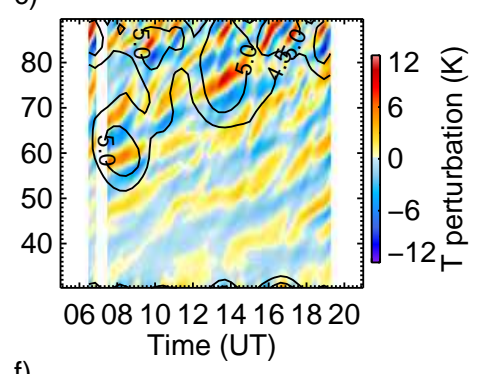

f)

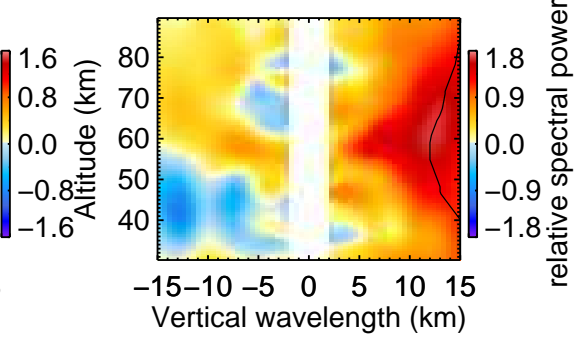

Figure 4: Gravity wave perturbations (a) $T^{\prime}(z, t)$, (b) $T_{+}^{\prime}(z, t)$, (c) $T_{-}^{\prime}(z, t)$ for 14 July 2014. The wavelet power is shown in contours in (b) and (c). Relative wavelet spectral power is shown as a function of (d) $\lambda_{z}$ vs. $c_{z}$, (e) $\lambda_{z}$ vs. $T$ and (f) $z$ vs. $\lambda_{z}$. Negative (positive) values on the x-axis indicate downward (upward) phase progression waves. The black curve in (f) marks the mean $\lambda_{z}(z)$. 


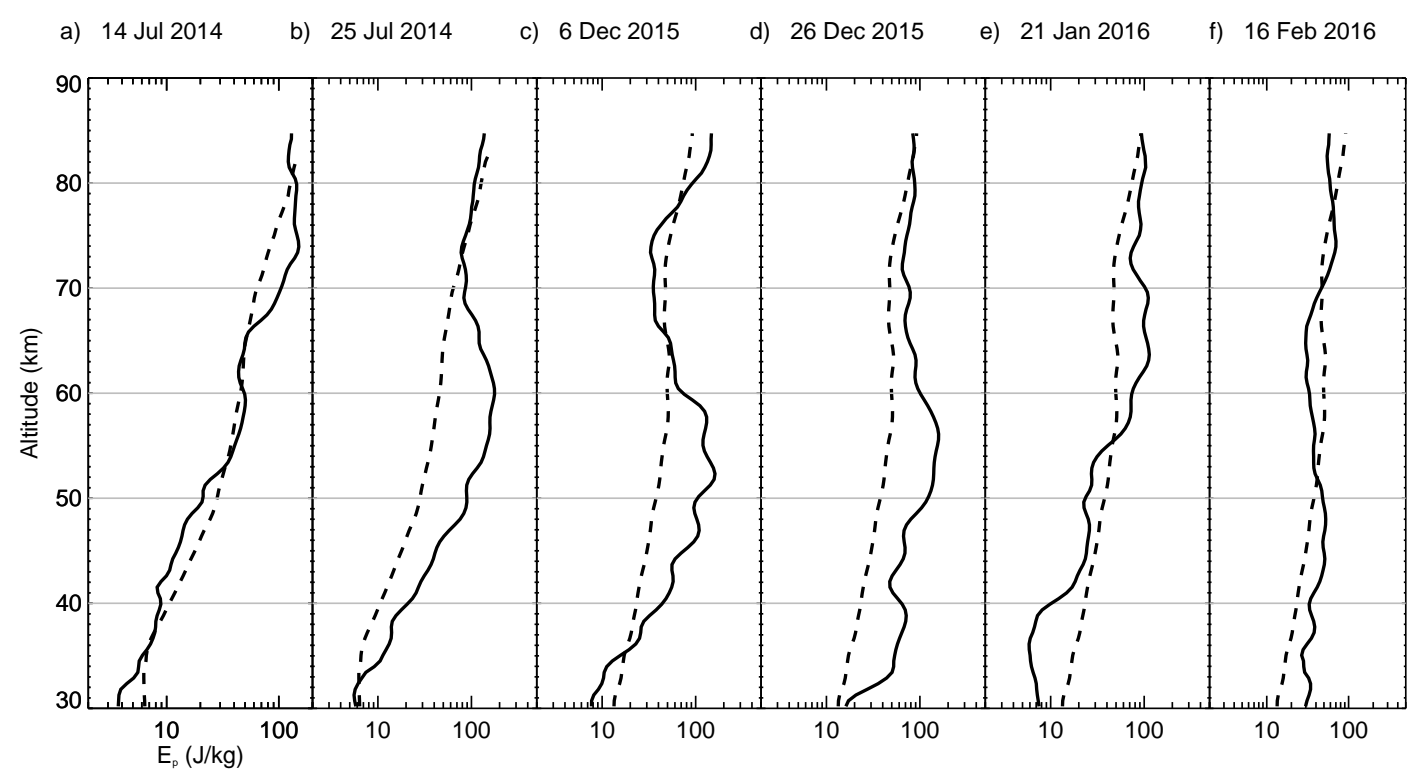

Figure 5: Potential energy densities for each of the six cases (a-f): total $E_{p}$ (black) and the dataset mean $E_{p, 0}$ (dashed) of the DEEPWAVE and GW-LCYCLE2 datasets, respectively. Profiles are smoothed with an $8 \mathrm{~km}$ moving average filter. 
$\begin{array}{llllll}\text { a) } 14 \mathrm{Jul} 2014 & \text { b) } 25 \mathrm{Jul} 2014 & \text { c) } 6 \mathrm{Dec} 2015 & \text { d) } 26 \mathrm{Dec} 2015 & \text { e) } 21 \text { Jan } 2016 & \text { f) } 16 \text { Feb } 2016\end{array}$

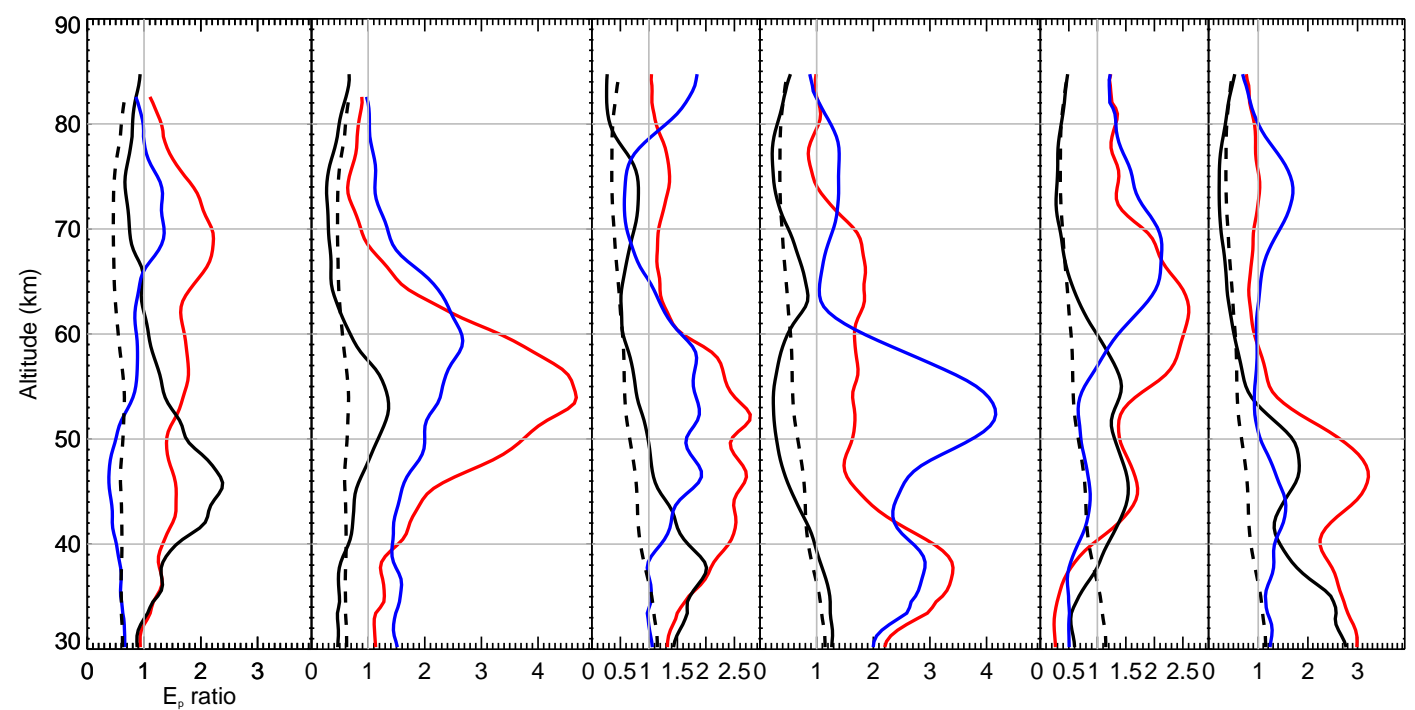

Figure 6: $E_{p}$ ratios for each of the six cases (a-f): $E_{\mathrm{p},+} / E_{\mathrm{p}, 0,+}$ (downward phase progression, blue), $E_{\mathrm{p},-} / E_{\mathrm{p}, 0,-}$ (upward phase progression, red), $E_{\mathrm{p},-} / E_{\mathrm{p},+}$ (black) and $E_{\mathrm{p}, 0,-} / E_{\mathrm{p}, 0,+}$ (black, dashed).

a)
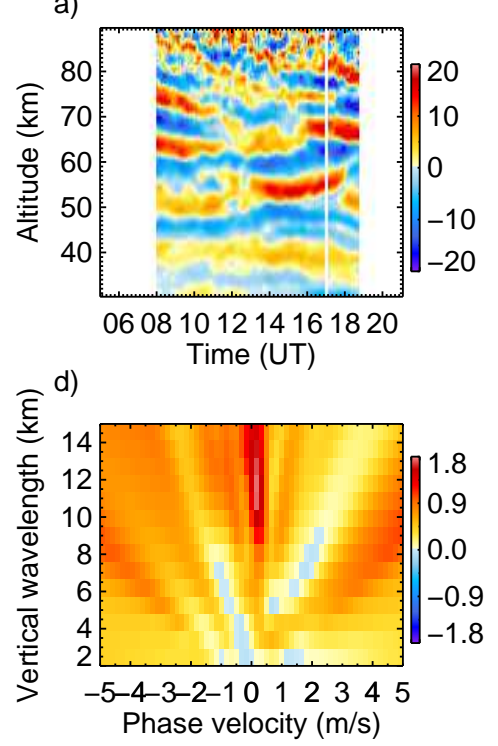

b)

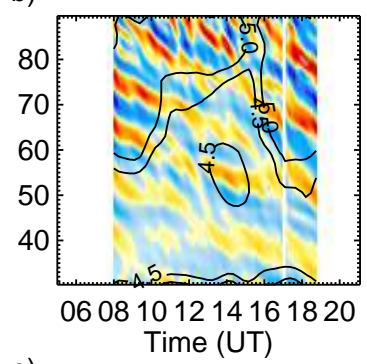

e)

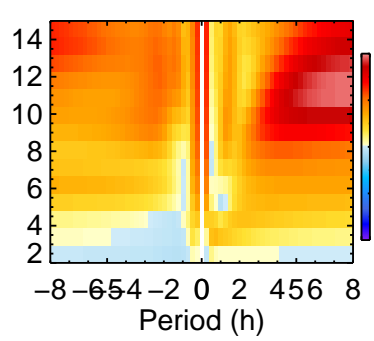

c)

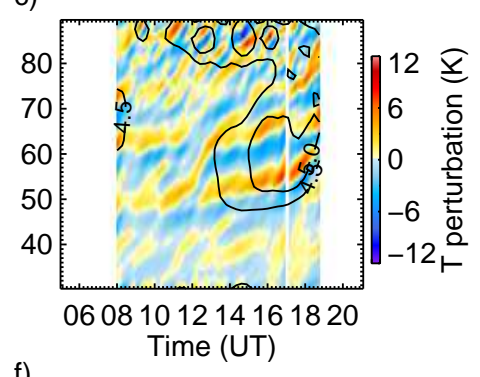

f)

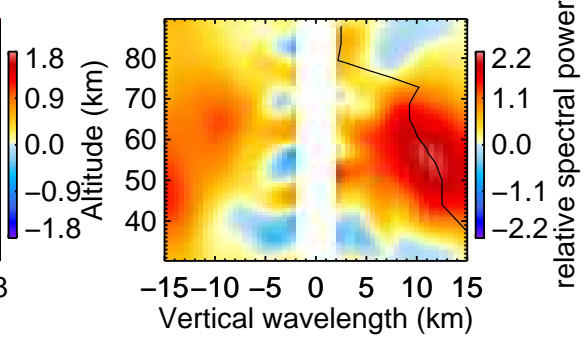

Figure 7: Same as Fig. 4 for 25 July 2014. 

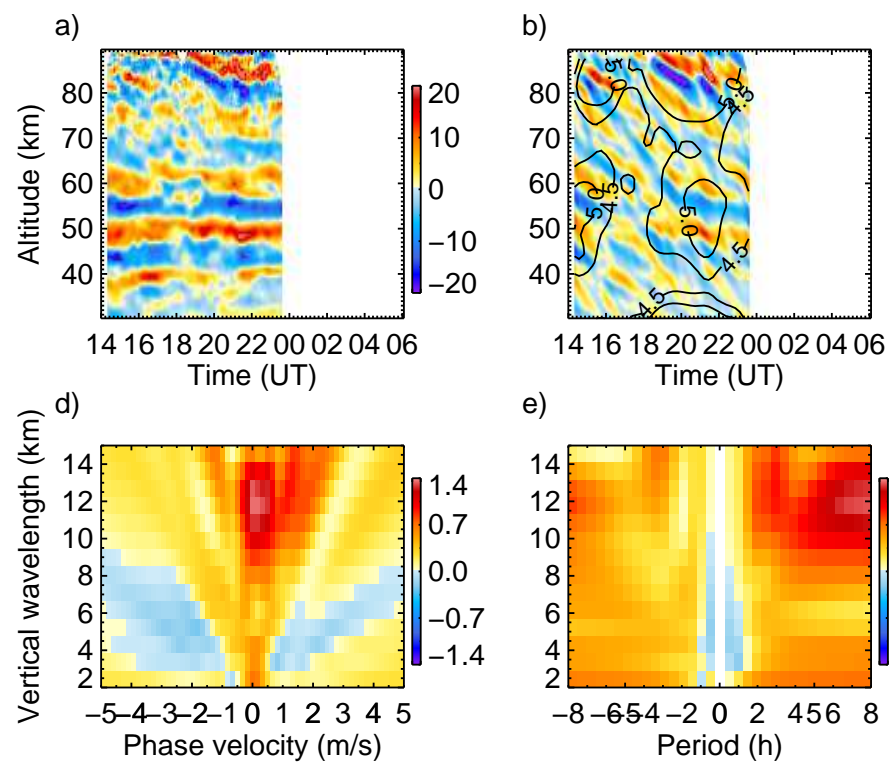

e)
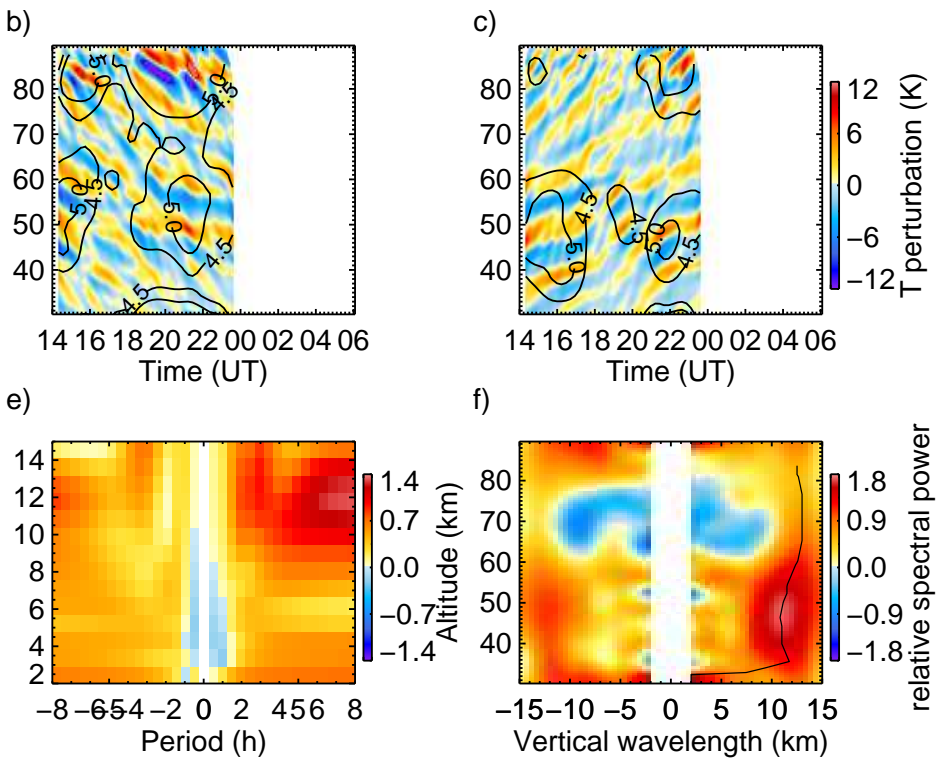

Figure 8: Same as Fig. 4 for 6 December 2015.

a)
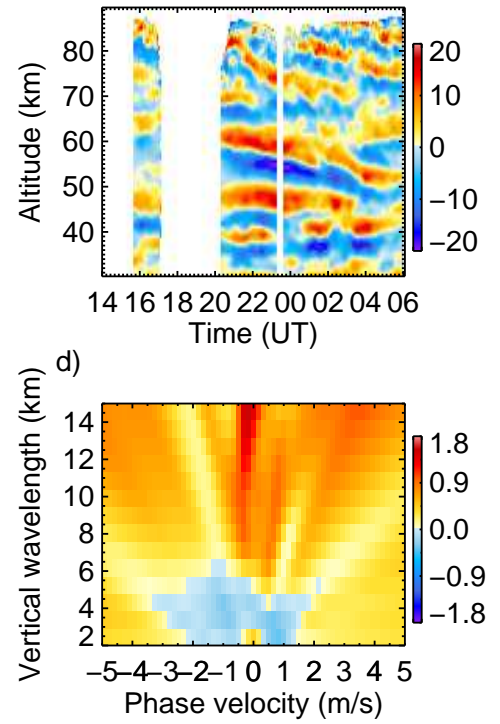

b)

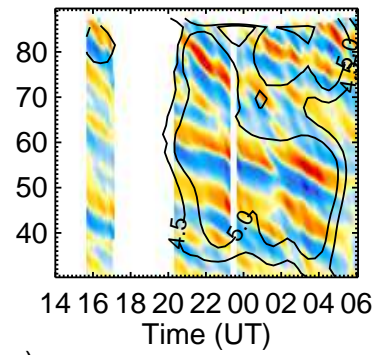

e)

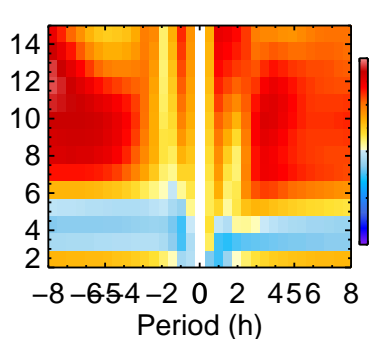

c)

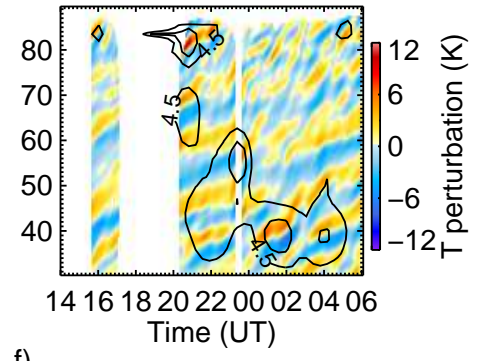

f)

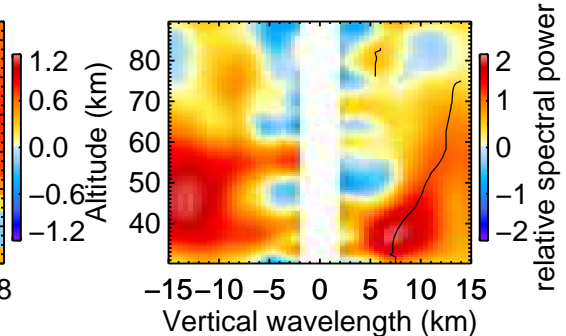

Figure 9: Same as Fig. 4 for 26 December 2015. 
a)
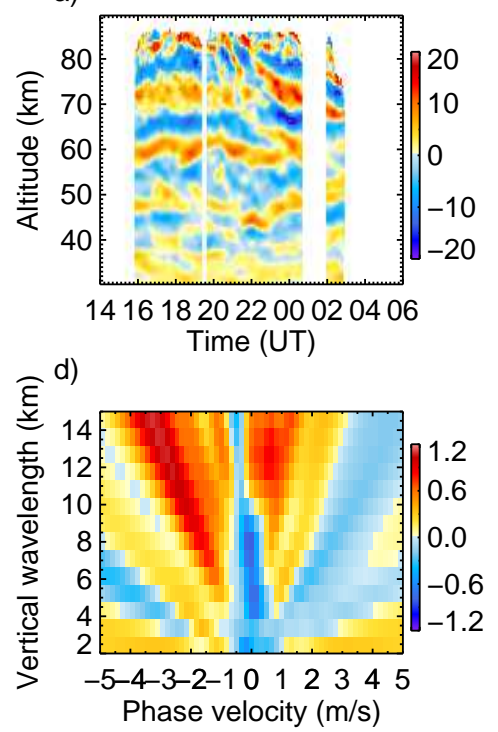

b)

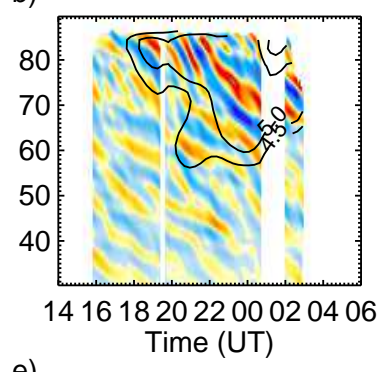

e)

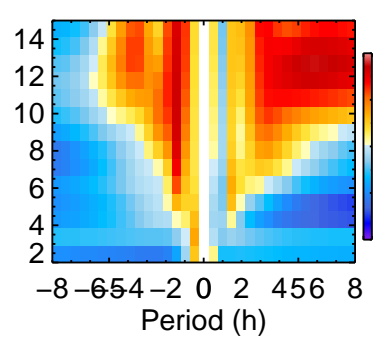

c)
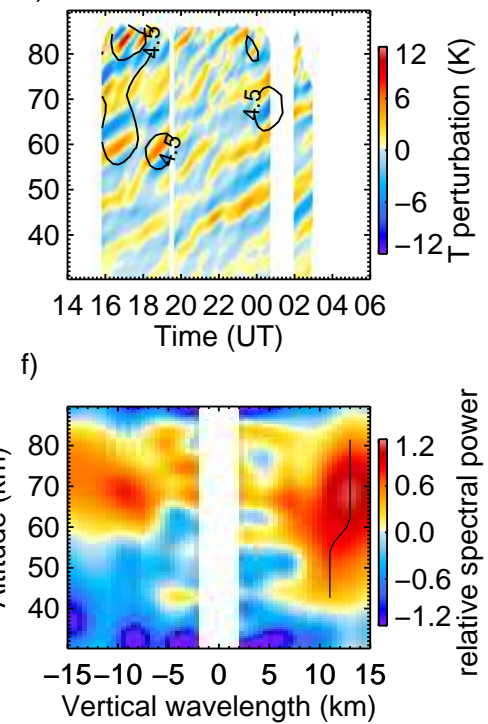

Figure 10: Same as Fig. 4 for 21 January 2016.

a)
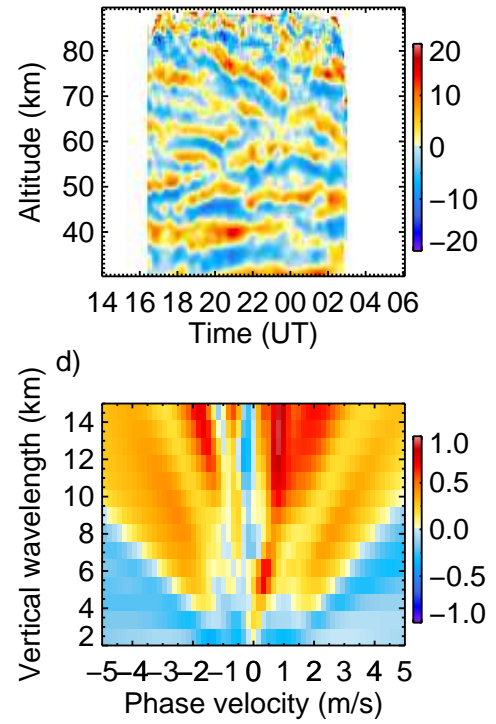

b)

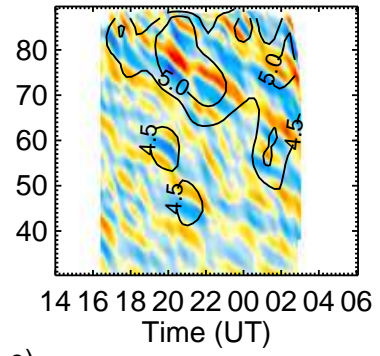

e)

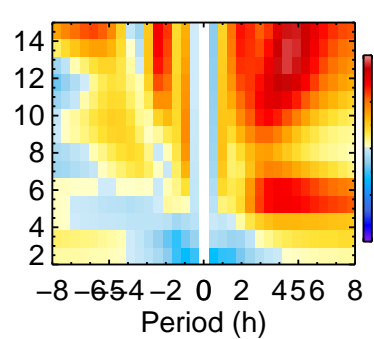

c)

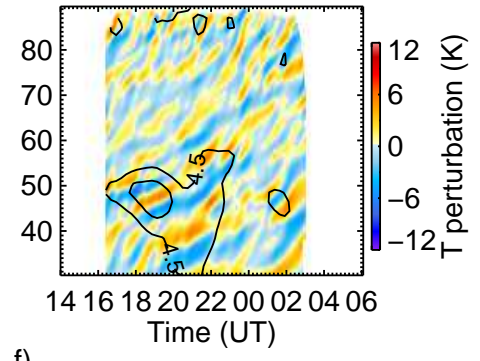

f)

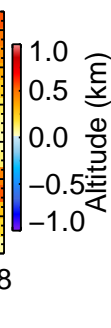

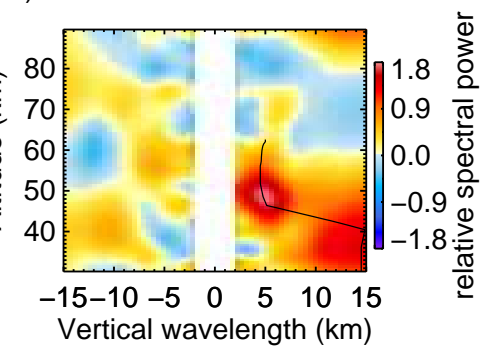

Figure 11: Same as Fig. 4 for 16 February 2016. 

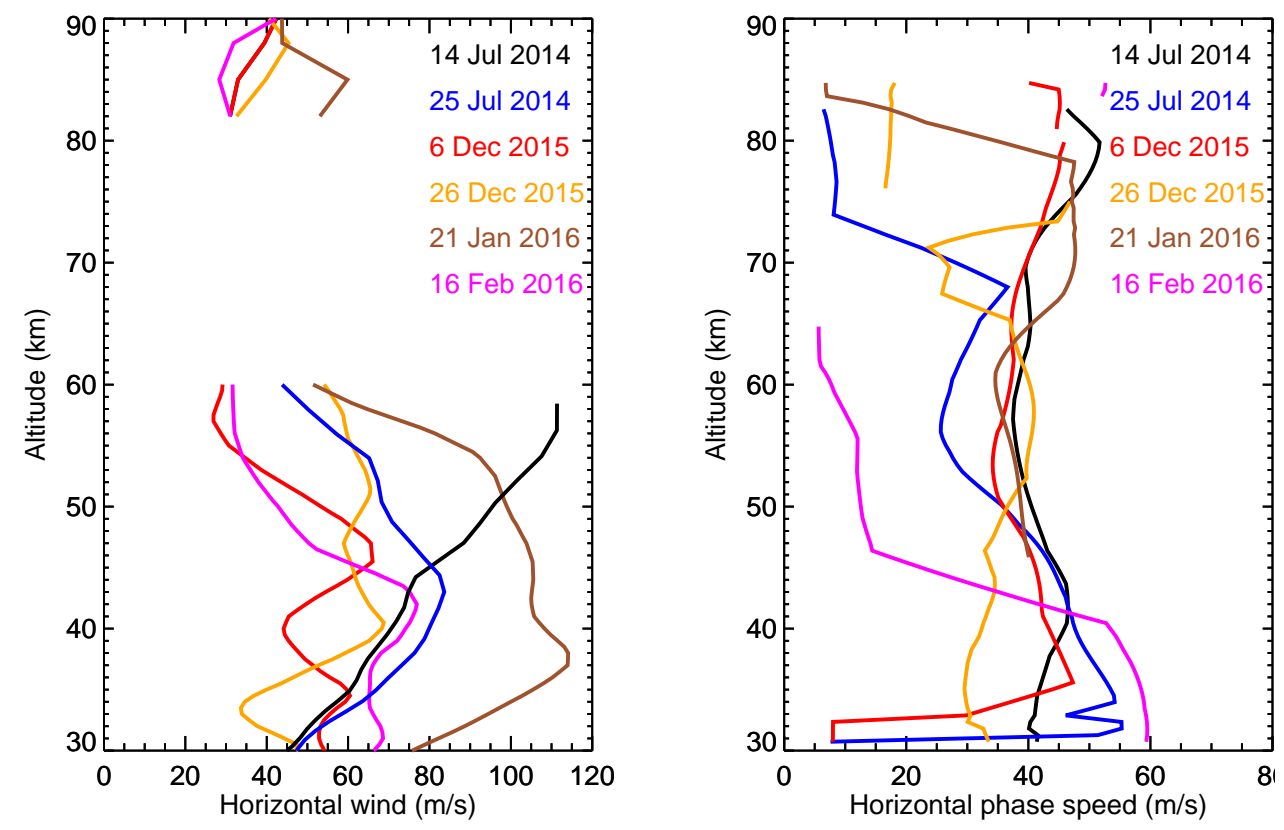

Figure 12: (a) Co-located horizontal wind data $\bar{u}_{h}$ from meteor radar measurements (above $82 \mathrm{~km}$ ) and ECMWF (below $60 \mathrm{~km}$ ). (b) Intrinsic horizontal phase velocity $\left|\hat{c}_{h}\right|$ of waves with upward phase progression. 